\title{
O sistema farmacêutico de inovação e o relacionamento empresa- universidade no setor farmacêutico do Estado do Rio de Janeiro*
}

\author{
Julia Paranhos ${ }^{1}$ \\ Lia Hasenclever ${ }^{2}$
}

\begin{abstract}
RESUMO
O Estado do Rio de Janeiro (ERJ) possui a segunda maior concentração de indústrias farmoquímicas e laboratórios farmacêuticos do país. Além disso, há um grande número de centros de pesquisas e universidades que podem ser importantes fontes de inovação para o desenvolvimento do setor regionalmente. O objetivo geral deste artigo é apresentar os resultados preliminares da análise sobre o sistema farmacêutico de inovação fluminense, considerando suas forças, fraquezas e os desafios que estão postos para o seu desenvolvimento, com especial ênfase ao estabelecimento das parcerias entre empresas e universidades. Os resultados apontam que, apesar da potencialidade do ERJ na produção do conhecimento na área e na formação de recursos humanos, a indústria farmacêutica fluminense vem apresentando um crescimento negativo desde meados dos anos 1990, com queda no número de estabelecimentos, número de empregos formais e produção física. O governo do estado vem buscando reverter este quadro negativo, mas as ações ainda são muito incipientes principalmente no que diz respeito à parceria empresa-universidade.
\end{abstract}

Palavras-chave: indústria farmacêutica; indústria farmoquímica; inovação; universidade-empresa; políticas públicas

\begin{abstract}
The state of Rio de Janeiro has the second largest concentration of pharmochemical industries and pharmaceutical laboratories in the country. Besides, there is a large number of research centers and universities that regionally can be important sources of innovation for the development of the sector. The general objective of this article is to present the preliminary results of the analysis about the pharmaceutical system of innovation of the region, considering the existing strengths, weaknesses and challenges for its development, with a special emphasis on the creation of partnerships between industry and university. The results point out that, in spite of the State's potentiality in the production of knowledge in the area and human resources formation, the pharmaceutical industry of Rio de Janeiro has been presenting a negative growth since

\footnotetext{
* As autoras agradecem aos bolsistas de iniciação científica Camila Serrano, Bruna Azevedo e Rainer Marques pelo apoio ao levantamento de dados e transcrição das entrevistas e à Faperj pelo apoio financeiro para o desenvolvimento dos dois projetos de pesquisa que geraram dados para este artigo.

${ }^{1}$ Doutora em Economia pelo Instituto de Economia da UFRJ. Professora Adjunta, Instituto de Economia, UFRJ.juliaparanhos@ufrj.br

${ }^{2}$ Doutora em Engenharia da Produção pela COPPE/UFRJ. Professora Associada, Instituto de Economia, UFRJ. lia@ie.ufrj.br
} 
the mid-1990's, with a fall in the number of units, number of formal employment and physical production. The State's government has been trying to change this negative situation but the actions taken are still too incipient, especially concerning the industryuniversity partnership.

Keywords: pharmochemical industry; pharmaceutical laboratory; innovation; university-industry; public policies

\section{Introdução}

A abordagem do sistema regional de inovação permite conhecer os processos de inovação em uma região. Ela ajuda a identificar os principais atores envolvidos no processo de aprendizado para gerar inovações bem como os obstáculos que devem ser resolvidos pelas instituições locais a fim de melhor interagirem. Lundvall et al. (2002) afirmam que a abordagem regional é complementar à abordagem dos sistemas nacionais de inovação.

Cooke et al. (1997) propõem a avaliação dos sistemas regionais de inovação sob duas perspectivas: uma enfatizando a questão da importância das políticas regionais para garantir a autonomia das regiões e outra do ponto de vista do potencial sistêmico, tal como o fortalecimento das interações entre as empresas e as universidades. $\mathrm{O}$ foco do artigo está baseado nestas duas perspectivas. Complementando esta abordagem, Fauré e Hasenclever (2007) reforçam a importância do local sobre o desenvolvimento das empresas, mas ressaltam que o desenvolvimento local é também "fruto das intervenções de fatores supralocais" (p. 15), devendo ser entendido como um fenômeno de múltiplas escalas. Com base nesta visão, apresenta-se uma análise sobre o desenvolvimento do setor farmacêutico no Estado do Rio de Janeiro (ERJ) durante os últimos 15 anos e as novas estratégias governamentais para sua melhor promoção.

Embora pioneiro na industrialização brasileira, o Rio de Janeiro passou por cerca de cinco décadas de consecutivos eventos - a transferência da capital para Brasília, a malograda fusão do Estado da Guanabara com o do Rio de Janeiro, a desindustrialização precoce, a migração de agências de publicidade e do setor financeiro para São Paulo e os impactos negativos da privatização sobre o mercado de trabalho local - que o levaram a perder sua importância no cenário nacional e sua posição de liderança industrial para o Estado de São Paulo (ESP) (Fauré e Hasenclever, 2005; Lessa, 2000). 
Os dados do crescimento econômico no ERJ mostram que este foi mais moderado do que a média nacional nos últimos anos. O PIB estadual cresceu a uma média anual de $2 \%$, enquanto em nível nacional o crescimento médio anual foi de $3 \%$, entre 1996 e 2008. A indústria de transformação, por exemplo, acumulou um decrescimento de 15,4\% no período de 1996 a 2008, enquanto em nível nacional a indústria de transformação acumulou um crescimento de 28,3\%. Por outro lado, a indústria extrativa do ERJ apresentou um desempenho extraordinário, acumulando um crescimento de $173 \%$, acima do crescimento nacional, que foi de $77 \%$. A elevada taxa de crescimento refere-se preponderantemente à expansão da atividade de extração do petróleo e de minério de ferro. Em 1996, a indústria extrativa respondia por 1,5\% do total da produção industrial. Em 2008, esse número elevou-se para 15,4\%. Já na indústria de transformação manteve sua participação em quase 10\% do PIB estadual. Mas, mesmo na indústria de transformação, o destaque ficou com as atividades de produção de coque e refino do petróleo, que representava $5,6 \%$ do valor bruto da produção industrial do total da indústria, em 1996, passando para 8\%, em 2007. Assim, verifica-se que o ERJ passou por um importante processo de mudança estrutural, predominando as atividades de serviços (inclusive comércio) e de produção industrial extrativa, que juntas representam atualmente $80 \%$ do PIB estadual. Esse destaque da indústria extrativa, porém, acende uma luz vermelha para a indústria fluminense, que está se especializando na extração de petróleo e minerais, enquanto outros setores industriais reduzem drasticamente sua produção, como foi o caso da indústria farmacêutica, cujo valor bruto da produção industrial acumulou um decréscimo de $42 \%$ (Hasenclever et al., 2012).

A redução da indústria de transformação no ERJ e as mudanças na esfera nacional no início da década de 1990 com forte impacto sobre o setor químicofarmacêutico (Paranhos, 2012) levaram à redução do setor no estado. É importante ressaltar que a ausência de políticas locais específicas para a promoção do setor farmacêutico, que tiveram início somente no final da década de 1990 de forma tímida e pontual, reforçaram o cenário negativo. Apesar disso, a indústria farmacêutica segue a tendência da indústria geral, concentrando-se em termos de unidades locais e volume de emprego principalmente na região Sudeste, e mais especificamente no ESP e no ERJ (Selan, Kannebley Jr. e Porto, 2007).

Em 2010, a economia do ERJ começa a mostrar sinais de recuperação alavancados por novas descobertas de petróleo. As ações do governo local para reduzir 
a violência e melhorar a imagem do estado no país e no mundo contribuíram também para atrair novos investimentos incluindo os Jogos Olímpicos de 2016 (Hasenclever et al., 2012; Urani e Giambiagi, 2011). Portanto, sob a visão da abordagem de desenvolvimento regional, neste momento é relevante identificar setores que tenham importante participação no cenário nacional e sejam também representativos no estado sendo o setor farmacêutico um deles - para melhorar a capacidade instalada e a interação empresa-universidade, assim como, para promover o sistema regional de inovação.

O artigo está dividido em quatro seções, além desta Introdução e da Conclusão. Na primeira seção é apresentada a metodologia da pesquisa. Na segunda seção, são analisados os dados secundários referentes à capacidade científica e de formação de recursos humanos, como também os dados de estabelecimento, emprego e produção física do setor farmacêutico no ERJ. Na terceira seção, são apresentadas as ações passadas e atuais do governo do ERJ para desenvolvimento do setor farmacêutico. E, por fim, na quarta seção, analisam-se os relatos da pesquisa de campo sobre a parceria empresa-universidade.

\section{Metodologia}

O objetivo geral do artigo é analisar o desempenho do sistema farmacêutico de inovação do Estado do Rio de Janeiro nos últimos 15 anos avaliando-se suas forças, fraquezas e os desafios para o seu desenvolvimento. Além disso, pretende-se também discutir as ações de seus atores para a promoção do desenvolvimento do setor de forma a gerar inovações, principalmente através da interação empresa-universidade. Ressaltam-se, assim, quais as vantagens existentes no ERJ capazes de reter os atores aqui localizados, quais as desvantagens que estes atores enfrentam e quais os desafios que o governo estadual precisa enfrentar para reduzir as desvantagens, aumentar as vantagens e promover a volta do crescimento e o desenvolvimento do setor no estado.

Este artigo apresenta os resultados de dois projetos de pesquisa financiados pela Fundação de Amparo à Pesquisa do Estado do Rio de Janeiro (FAPERJ) pelo edital Cientista do Nosso Estado (Hasenclever, 2012) e Auxílio à Pesquisa (Paranhos, 2011), este último ainda em andamento. Neste sentido, as informações nele apresentadas combinam os resultados sobre a interação empresa-universidade do primeiro projeto e os resultados preliminares do segundo. 
A metodologia utilizada foi um estudo de caso desenvolvido a partir de revisão da literatura sobre o desenvolvimento regional e dos sistemas de inovação (Cooke et al., 1997; Lundvall et al., 2002, Fauré e Hasenclever, 2005 e 2007) sobre o setor farmacêutico nacional (Bermudez et al., 2000; Buss et al., 2008; Hasenclever, 2002; Paranhos, 2012) e sobre o desenvolvimento do ERJ (Hasenclever et al., 2012; Lessa, 2000; Urani e Giambiagui, 2011). Realizou-se ainda uma análise de dados secundários (Relação Anual de Informações Sociais do Ministério do Trabalho e Emprego RAIS/MTE; Pesquisa Industrial Mensal de Produção Física do Instituto Brasileiro de Geografia e Estatística - PIM-PF/IBGE) e pesquisa de campo para obtenção de dados primários.

$\mathrm{Na}$ pesquisa de campo (realizada para os dois projetos supracitados), foram realizadas entrevistas com 23 importantes atores do setor farmacêutico no estado entre 2008 e 2012, dentro de uma pesquisa mais ampla em nível nacional. Entre os entrevistados estão empresas nacionais públicas e privadas, empresas multinacionais, instituições do governo estadual e federal (agências de fomento, de regulação e formuladores de política), pesquisadores e agências de inovação de universidades e institutos de pesquisa, associações empresariais e atores ligados ao setor. Neste sentido, os resultados obtidos são uma compilação dos principais fatores identificados pelos atores do próprio setor. As entrevistas foram presenciais, baseadas em formulários com perguntas abertas e fechadas. Para garantia do anonimato do entrevistado e de sua instituição no relato das entrevistas, os mesmos são identificados por códigos de acordo com a Tabela 1. Como o projeto está em andamento, outras entrevistas ainda estão sendo realizadas, mas seus resultados não foram incluídos neste artigo.

\section{Tabela 1. Códigos dos entrevistados da pesquisa de campo}

\begin{tabular}{l|c|}
\hline \multicolumn{1}{c|}{ Entrevistados } & Código \\
\hline Empresas farmacêuticas nacionais & EFN \\
\hline Empresas farmacêuticas multinacionais & EFM \\
\hline Laboratórios públicos oficiais & LPO \\
\hline Empresas de insumo farmacêutico & EIF \\
\hline CROs nacionais & CRO \\
\hline Empresas de pesquisa farmacêutica & EPF \\
\hline Pesquisadores & PES \\
\hline Agências de inovação & AIU \\
\hline Incubadora de empresa & IEB \\
\hline Instituições do governo & GOV \\
\hline Outros atores ligados ao setor & OAS \\
\hline
\end{tabular}

Fonte: Pesquisa de Campo, Elaborado pelo GEI/IE/UFRJ. 


\section{Panorama do setor farmacêutico no Estado do Rio de Janeiro}

O ERJ aparece no cenário nacional como o segundo estado em termos de concentração de estabelecimentos e empregos no setor farmacêutico, atrás somente do ESP. A especialização do ERJ no setor está presente principalmente na capital, com 50 dos 75 estabelecimentos fabricantes de produtos farmacêuticos do estado e 16 dos 23 estabelecimentos de produtos farmoquímicos (RAIS/MTE, 2010). Outros municípios com estabelecimentos neste setor são Campos dos Goytacazes, Duque de Caxias, Guapimirim, Itatiaia, Macaé, Niterói, Nova Iguaçu, Paraíba do Sul, Resende, São Gonçalo, São João de Meriti, Saquarema, Teresópolis, Três Rios, Valença e Volta Redonda.

Esta característica de forte concentração na capital é uma marca do estado nos seus diversos setores e atividades, como pode ser notado também nos dados referentes às instituições científicas e tecnológicas (ICTs). O ERJ possui significativa especialização nas áreas do conhecimento relacionadas ao setor farmacêutico demonstrando uma forte capacidade científica e de formação de recursos humanos, como apresentado em Hasenclever (2012). São ao todo 39 ICTs públicas e privadas, das quais três são universidades estaduais (UERJ, UENF e UEZO) ${ }^{3}$ e quatro são universidades federais (UFRJ, UFF, UFRRJ, Unirio). ${ }^{4}$ Destas sete universidades, somente três não estão localizadas na cidade do Rio de Janeiro, mas em Niterói (UFF), Seropédica (UFRRJ) e Campos de Goytacazes (UENF). Tem-se um total de 158 cursos de graduação nas grandes áreas do conhecimento relacionadas ao setor farmacêutico: Ciências Biológicas (50\%), Ciências Biológicas I (7\%), Farmácia (22\%), Química (19\%) e Química Industrial (2\%), conforme a Tabela 2 abaixo - cenário este também considerado muito significativo por Britto et al. (2011).

\footnotetext{
${ }^{3}$ Universidade Estadual do Rio de Janeiro, Universidade Estadual do Norte Fluminense e Universidade Estadual da Zona Oeste.

${ }^{4}$ Universidade Federal do Rio de Janeiro, Universidade Federal Fluminense, Universidade Federal Rural do Rio de Janeiro, Universidade Federal do Estado do Rio de Janeiro.
} 
Tabela 2. Cursos de graduação no setor farmacêutico

\begin{tabular}{|l|c|}
\hline \multicolumn{1}{|c|}{ ÁREA DO CONHECIMENTO } & $\begin{array}{c}\mathbf{N}^{\mathbf{0}} \text { DE CURSOS } \\
\text { PRESENCIAIS }\end{array}$ \\
\hline CIÊNCIAS BIOLÓGICAS & 79 \\
\hline CIÊNCIAS BIOLÓGICAS I $(*)$ & 11 \\
\hline FARMÁCIA & 35 \\
\hline QUÍMICA & 30 \\
\hline QUÍMICA INDUSTRIAL & 3 \\
\hline \multicolumn{1}{|c|}{ TOTAL DE CURSOS } & $\mathbf{1 5 8}$ \\
\hline
\end{tabular}

(*) CIÊNCIAS BIOLÓGICAS I: BIOFÍSICA, BIOLOGIA MARINHA, BIOLOGIA VEGETAL, BIOTECNOLOGIA, ECOLOGIA, GENÉTICA MICROBIOLOGIA E IMUNOLOGIA, MODALIDADE MÉDICA, ZOOLOGIA, FÍSICA E QUÍMICA.

Fonte: MEC (2012).

Também em termos de pós-graduação a capacitação do estado se mantém (Hasenclever, 2012). Estão presentes no ERJ 10 ICTs, sendo duas universidades estaduais (UERJ, UENF), quatro universidades federais (UFRJ, UFF, UFRRJ, Unirio), três institutos federais de pesquisa (Fiocruz, IME, IBRJ) ${ }^{5}$ e uma universidade privada (PUC-RIO). ${ }^{6}$ Na pós-graduação, repete-se a concentração das ICTs na cidade do Rio de Janeiro, e somente as três mencionadas acima não estão localizadas na capital. Nestes ICTs, há 39 mestrados, 3 mestrados profissionais e 35 doutorados nas áreas do conhecimento relacionadas ao setor farmacêutico mostradas na Tabela 3. Percebe-se grande concentração dos cursos nas áreas de Ciências Biológicas I, com 33\% dos cursos de mestrado e $31 \%$ de doutorado, e Ciências Biológicas II, com $26 \%$ dos cursos de mestrado e $29 \%$ de doutorado, além dos $67 \%$ do mestrado profissional. No que diz respeito à pesquisa, o estado conta com um total de 307 grupos de pesquisa em Biologia (45\%), Química (43\%), Farmácia (11\%) e Medicina (1\%), segundo o DP-CNPq (CNPq, 2010).

\footnotetext{
${ }^{5}$ Fundação Oswaldo Cruz, Instituto Militar de Engenharia, Instituto de Pesquisas Jardim Botânico do Rio de Janeiro.

${ }^{6}$ Pontifícia Universidade Católica.
} 
Tabela 3. Cursos de pós-graduação no setor farmacêutico

\begin{tabular}{|c|c|c|c|c|}
\hline \multicolumn{2}{|c|}{ CARACTERÍSTICAS } & \multicolumn{2}{c|}{$\mathbf{N}^{\mathbf{0}}$ DE CURSOS DE PÓS-GRADUAÇÃO } \\
\cline { 2 - 5 } & MESTRADO & $\begin{array}{c}\text { MESTRADO } \\
\text { PROFISSIONAL }\end{array}$ & DOUTORADO \\
\hline \multirow{4}{*}{$\begin{array}{c}\text { ÁREA DO } \\
\text { CONHECIMENTO }\end{array}$} & $\begin{array}{c}\text { CIÊNCIAS BIOLÓGICAS I } \\
(*)\end{array}$ & 13 & 0 & 2 \\
\cline { 2 - 5 } & $\begin{array}{c}\text { CIÊNCIAS BIOLÓGICAS II } \\
(* *)\end{array}$ & 10 & 2 & 11 \\
\cline { 2 - 5 } & $\begin{array}{c}\text { CIÊNCIAS BIOLÓGICAS III } \\
(* * *)\end{array}$ & 4 & 0 & 10 \\
\cline { 2 - 5 } & FARMÁCIA & 2 & 1 & 3 \\
\cline { 2 - 5 } & QUÍMICA & 8 & 0 & 1 \\
\hline \multirow{2}{*}{ INSTITUTIÇÃO } & PÚBLICA & 38 & 3 & 34 \\
\cline { 2 - 5 } & PRIVADA & 1 & 0 & 3 \\
\hline \multicolumn{2}{|c|}{ TOTAL DE CURSOS } & $\mathbf{3 9}$ & $\mathbf{3}$ & $\mathbf{3 5}$ \\
\hline
\end{tabular}

(*) CIÊNCIAS BIOLÓGICAS I: BIOLOGIA GERAL, BOTÂNICA, GENÉTICA, OCEANOGRAFIA BIOLÓGICA, ZOOLOGIA

(**) CIÊNCIAS BIOLÓGICAS II: BIOFÍSICA, BIOLOGIA MOLECULAR, BIOQUÍMICA, FARMACOLOGIA, FISIOLOGIA, MORFOLOGIA (***) CIÊNCIAS BIOLÓGICAS III: MICROBIOLOGIA, MICROBIOLOGIA APLICADA, PARASITOLOGIA

Fonte: Capes (2011).

No entanto, em termos produtivos, como também apresentado em Hasenclever (2012), o setor farmacêutico apresenta forte queda nos últimos 15 anos conforme podese perceber na análise dos dados da RAIS/MTE e da PIM-PF/IBGE apresentados a seguir. Pelos dados de número de estabelecimentos apresentados no Gráfico 1, percebese uma redução significativa na participação do número de estabelecimentos do setor farmacêutico no ERJ no total do país. Em 1995, localizavam-se no estado cerca de $19,8 \%$ dos estabelecimentos do setor farmacêutico do país, compreendendo os estabelecimentos produtores de farmoquímicos e de medicamentos para uso humano. No entanto, no final do período analisado, 2010, este percentual chega a atingir apenas 13,6\%. Esta queda de 6 pontos percentuais (p.p.) ganha maior importância quando comparada à queda de 3 p.p. do ESP e o forte crescimento de mais de 5 p.p. do Estado de Goiás (EGO). Importante ressaltar que estas tendências ocorrem também em números absolutos, com uma redução no número de estabelecimentos farmacêuticos no ERJ de $46 \%$ e no ESP de $28 \%$ nos 15 anos e um crescimento de $156 \%$ no EGO. 


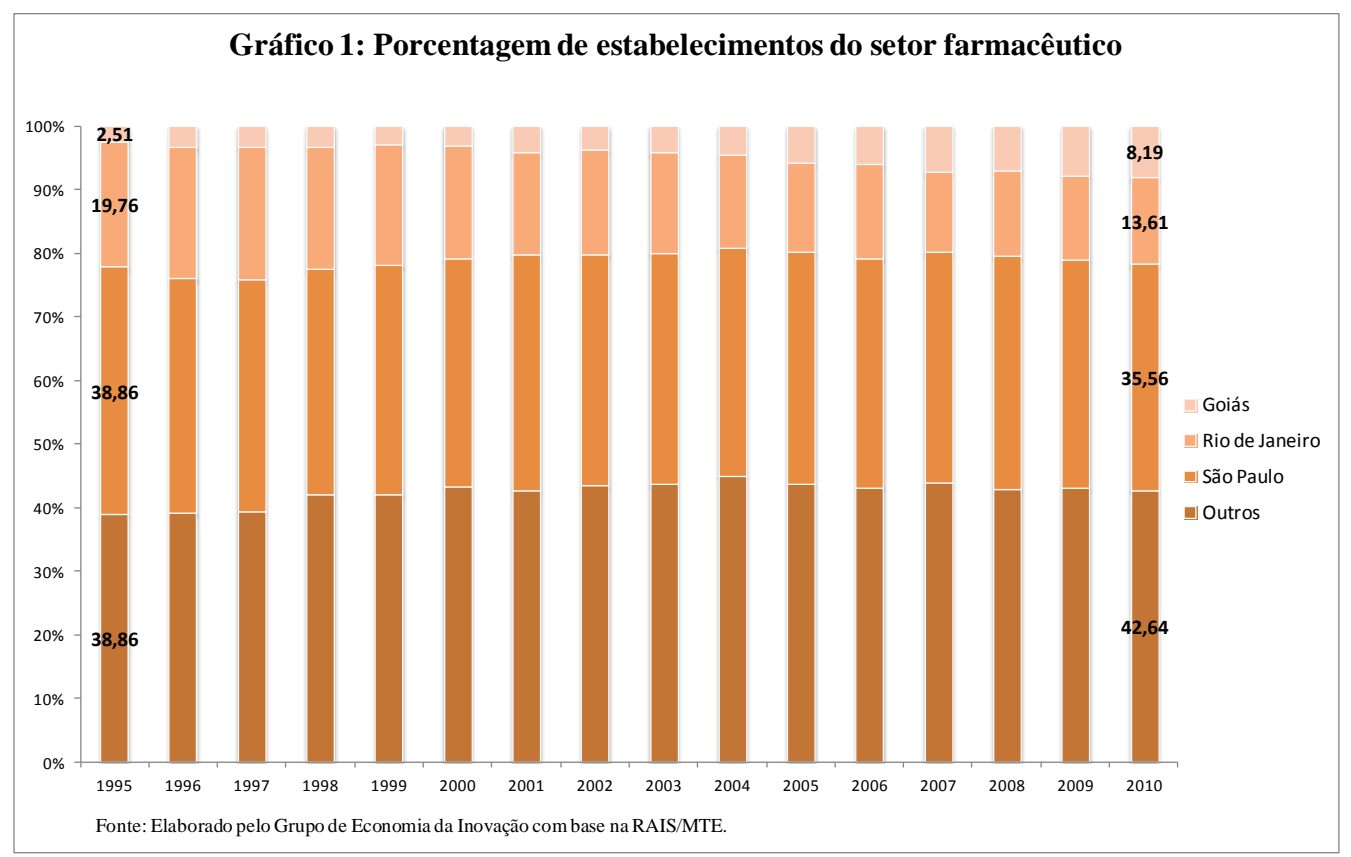

Através da RAIS/MTE também foi possível obter a quantidade de trabalhadores formais que estão empregados nestes estabelecimentos. É importante ressaltar uma queda ainda mais expressiva da que ocorre com os estabelecimentos. Em 1995, 21,9\% de trabalhadores estavam empregados no setor farmacêutico do ERJ, porém em 2010 este percentual diminui para 9,6\%, acarretando uma alta queda de emprego formal no setor de mais de 12 p.p.. O ESP também apresentou queda, ainda que bem menor (4 p.p.), mas o EGO cresceu quase 3 vezes sua participação no emprego formal do setor farmacêutico do país, e o grupo outros, com os demais estados do país, apresentou crescimento de 8 p.p. - o que demonstra uma desconcentração do emprego no setor farmacêutico, com perda significativa do ERJ. Quando analisados os números absolutos do emprego no setor farmacêutico, percebe-se a redução de $36 \%$ no ERJ, o aumento de $435 \%$ no EGO e o crescimento no ESP de 36\% entre 1995 e 2010. Ou seja, o ESP perdeu participação, mas não teve queda no número de empregados, enquanto o ERJ perdeu participação e reduziu o número de empregados do setor farmacêutico. 


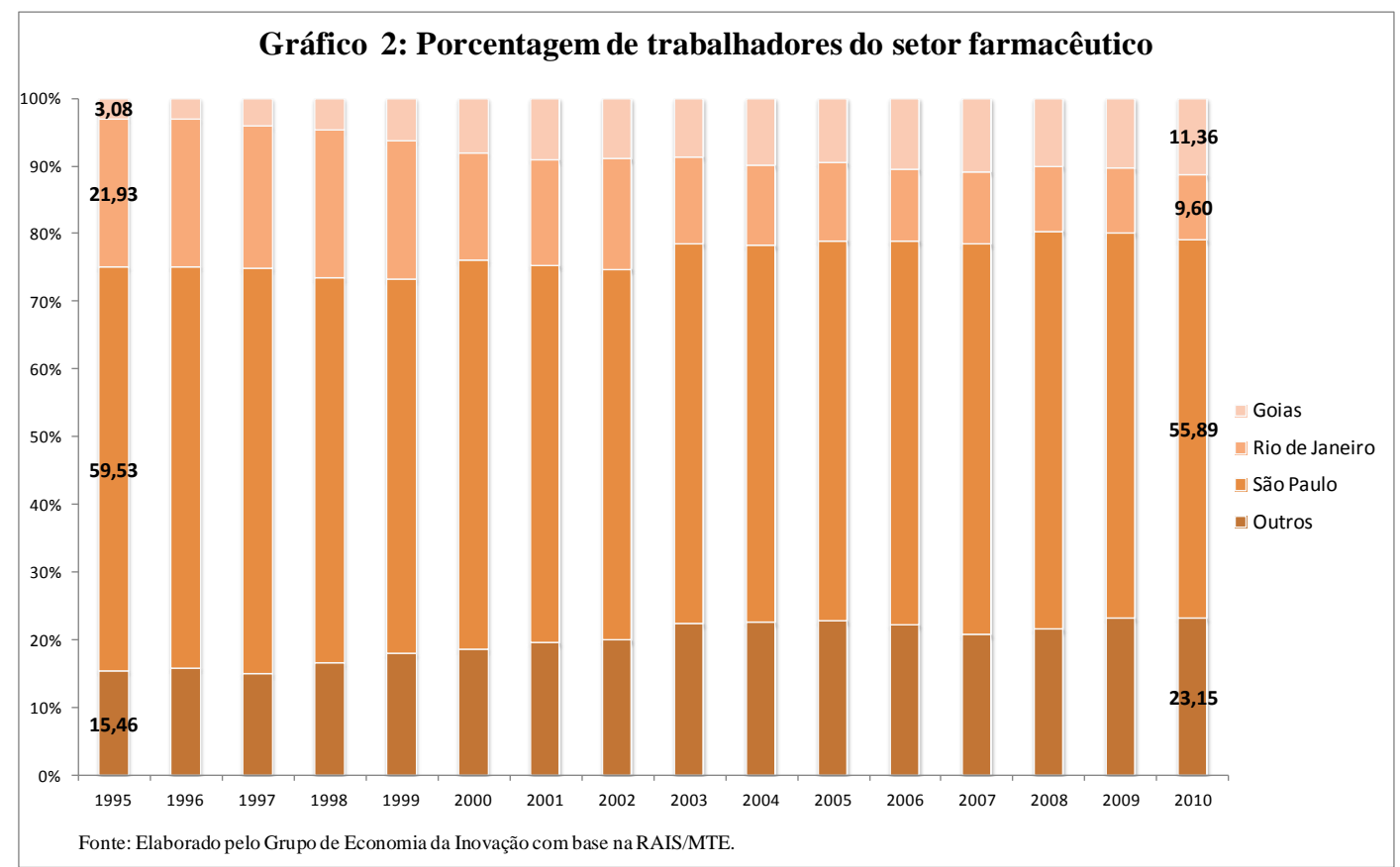

Vale observar ainda que a queda no número de trabalhadores foi acompanhada não somente pela redução no número de estabelecimentos, mas também pela queda significativa na produção física do setor farmacêutico no ERJ, de acordo com os dados da PIM-PF/IBGE. Para ilustrar esta queda, apresentam-se no Gráfico 3 os índices de produção física do setor farmacêutico e da indústria de transformação do ERJ e do Brasil no período de 1996 a 2010, com base no ano de 1996.

Gráfico 3. Produção física da indústria de transformação e farmacêutica no Brasil e ERJ

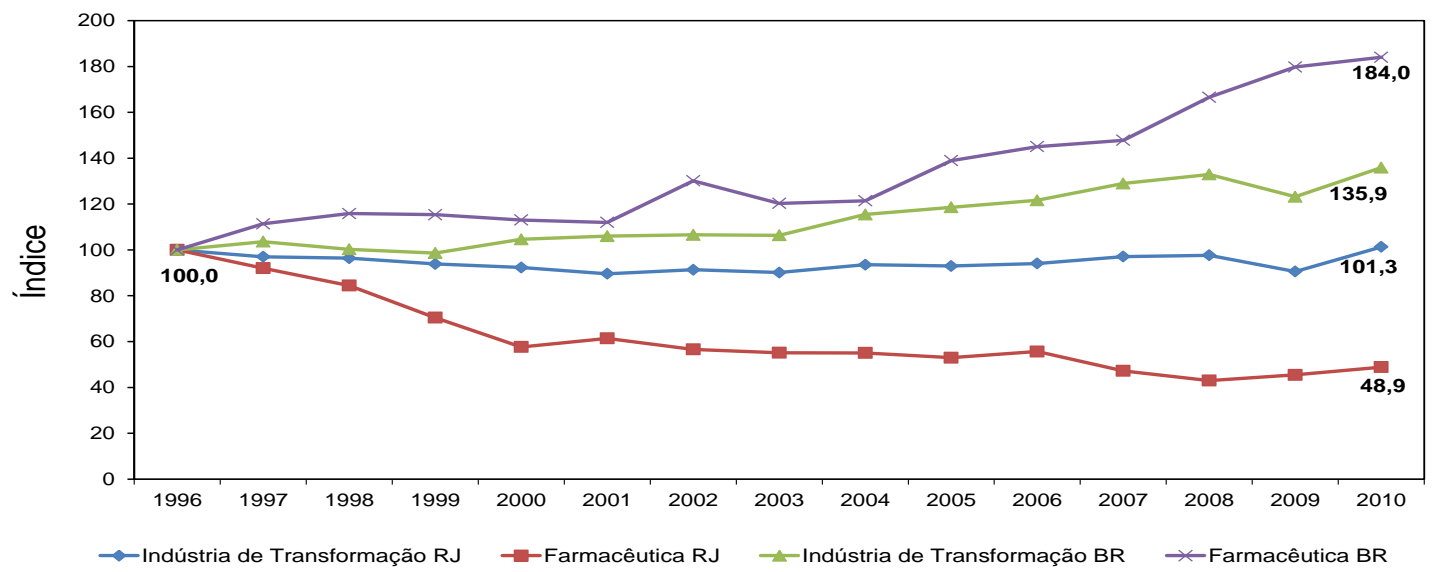

Fonte: Elaborado pelo Grupo de Economia da Inovação com base PIM-PF/IBGE. 
O curioso de se constatar é que a relação entre a produção física das indústrias farmacêuticas e de transformações no ERJ, durante o período em questão, é inversa ao comportamento das mesmas em âmbito nacional. No ERJ, a indústria farmacêutica apresenta queda na produção física, chegando a 57\% negativos em 2008, o índice mais baixo no período analisado, e 51,1\% negativos em 2010 - valores bastante inferiores aos da indústria de transformação fluminense que não apresenta uma evolução muito positiva, mas mantém-se estável.

Por outro lado, o crescimento da produção física da indústria farmacêutica do Brasil é superior à produção física da indústria de transformação no país durante todo o período analisado. A indústria de transformação no nível nacional apresenta crescimento de quase $36 \%$ em 2010, enquanto a produção física da indústria farmacêutica apresenta índices ainda superiores, chegando a $84 \%$ de crescimento em 2010 em relação a 1996 - o que revela a grande importância que a indústria farmacêutica tem para o país, como um setor dinâmico, que vem crescendo a cada ano.

\section{Políticas de apoio ao setor farmacêutico no ERJ}

Portanto, dos dados apresentados na seção anterior, é possível verificar que, apesar da grande capacidade científica e de formação de recursos humanos, a configuração do setor farmacêutico fluminense vem se alterando com a perda de muitos estabelecimentos e trabalhadores que antes faziam parte do conjunto farmacêutico atuante no estado. A partir desta realidade, cabe destacar quais foram as principais ações do governo estadual no intuito de reverter tal situação. Para isso, é apresentado a seguir um levantamento das principais ações do governo estadual nos últimos anos conforme Hasenclever (2012).

O principal foco de ação do governo do ERJ foi a implantação de incentivos fiscais às atividades de produção e comercialização do setor farmacêutico com o intuito de manter as empresas instaladas e a atrair novas empresas. Em 1998, foi estabelecido o Programa Rio Fármacos disposto no Decreto n ${ }^{\circ} 24.857 / 98$. O programa setorial prevê o desenvolvimento da indústria química fina de aplicações biotecnológica, farmacêutica, de fármacos e de cosméticos. É um programa de fomento à cadeia farmacêutica, que concede tratamento tributário especial para os estabelecimentos industriais, atacadistas e distribuidores integrantes da cadeia farmacêutica, oferece ainda uma linha de crédito de até $200 \%$ do valor do investimento fixo a ser liberada em parcelas mensais durante os 
cinco anos de operação das novas instalações. São também considerados investimentos fixos as despesas incorridas pelas empresas em testes de bioequivalência e biodisponibilidade, necessários para a produção de medicamentos genéricos.

Alguns anos depois, em 2004, um novo decreto com incentivos à redução do imposto sobre circulação de mercadorias e serviços (ICMS) foi implantado através do Decreto $n^{\circ} 36.450 / 04$ que oferece redução de 4\% de crédito presumido sobre o valor da nota fiscal e redução da base de cálculo do ICMS para 12\%, ficando assim a carga tributária reduzida em $8 \%$ para empresas da cadeia farmacêutica. No caso da importação, se o desembaraço aduaneiro ocorrer nos portos ou aeroportos fluminenses, o Decreto também dispõe sobre o deferimento do ICMS referente a esta ou à aquisição interna de insumos e bens para o ativo fixo.

No que diz respeito à inovação, cabe destacar a Lei de Inovação Estadual $n^{\circ}$ 5.361/08 e o Decreto $\mathrm{n}^{\circ}$ 42.302/10 que a regulamenta, que têm como objetivo incentivar a inovação e a pesquisa científica e tecnológica nos setores produtivos do ERJ. É possível observar ainda que o governo tem a intenção de priorizar em suas compras a aquisição de produtos desenvolvidos com base nessa lei.

Outra forma de ação do governo estadual tem sido o financiamento a projetos através das agências de fomento estaduais: a Faperj e Fundação de Apoio à Escola Técnica (Faetec). Estas agências, através do lançamento de editais, incentivam atividades científicas, tecnológicas e de inovação, apoiando instituições de ensino e/ou pesquisa, assim como projetos à inovação tecnológica de empresas sediadas no estado.

Acrescentam-se a estes programas as recentes ações do governo estadual a partir de 2010, em especial da Secretaria de Desenvolvimento Econômico Energia Indústria e Serviços (Sedeis), com objetivo de mudar o rumo do setor farmacêutico no estado e fazer com que este volte a apresentar números positivos de crescimento. Em 2011, após uma série de seminários e debates, ${ }^{7}$ um conjunto de importantes atores do setor farmacêutico no ERJ ${ }^{8}$ com o apoio da Sedeis cria o Grupo Executivo do Complexo Industrial em Ciências da Vida do Rio de Janeiro (GECIV-RJ) com a finalidade de elaborar e desenvolver as diretrizes das políticas estaduais de fortalecimento do complexo produtivo e de inovação em ciências da vida (Decreto estadual $\mathrm{n}^{\circ}$

\footnotetext{
7 "Diretrizes para uma Política de Desenvolvimento do Setor Químico-Farmacêutico do Estado do Rio de Janeiro" em 19/03/2010, "Diretrizes para uma Política de Desenvolvimento dos Setores de Biotecnologia e Químico-Farmacêutico do Estado do Rio de Janeiro" em 12/04/2011 e "Workshop Investe Rio: O que falta para o Estado do Rio tornar-se o locus da Biotecnologia?" em 17/10/2011.

${ }^{8}$ Investe Rio, Instituto Vital Brazil, BioRio, Rede de Tecnologia do Rio de Janeiro, Universidade Estadual do Rio de Janeiro entre outros.
} 
43.315/2011). Neste momento, parece iniciar no ERJ um período de maior atenção das políticas públicas para o setor farmacêutico, neste caso com maior foco em produtos biotecnológicos.

Em 2013, sai pela Faperj o primeiro edital pensado, discutido e elaborado no âmbito do GECIV-RJ para apoio aos projetos de pesquisa, desenvolvimento e inovação biotecnológica em saúde humana no Estado do Rio de Janeiro, voltado para financiar a execução de projetos que objetivem a realização de testes pré-clínicos ou clínicos, nas fases I, II e III.

\section{A parceria empresa e universidade no sistema farmacêutico de inovação brasileiro e fluminense}

Em termos do relacionamento empresa-universidade, os resultados da pesquisa de campo no estado apresentam o mesmo padrão nacional, com empresas pouco estruturadas em suas atividades inovativas e com grande foco na produção de medicamentos genéricos; e com universidades sem preparo para interagir com o setor empresarial, em especial, devido a sua rígida estrutura interna e às dificuldades de funcionamento dos núcleos de inovação tecnológica (NITs). Tais características, tanto das empresas farmacêuticas quanto das universidades, parecem indicar que elas não estão preparadas do ponto de vista de seu posicionamento estratégico e de sua missão para inovarem e contribuírem para o desenvolvimento local, exatamente como era de se esperar em um país onde a criação de ambas se deu de forma paralela. A indústria, criada a partir da importação de tecnologia, prescindiu da universidade para gerar e transferir conhecimentos (Suzigan e Albuquerque, 2011).

No que diz respeito ao tipo de atividades colaborativas realizadas entre empresas e universidades, a maioria são testes, que dependem dos equipamentos da universidade e do conhecimento técnico já desenvolvido em seu interior. Isto significa que pouco conhecimento novo está sendo criado nestas atividades, pois são atividades específicas realizadas aleatoriamente e por um breve período de tempo sem o envolvimento de muito conhecimento e pesquisa novos, isto é, que estejam criados e desenvolvidos para tal. É interessante atentar para o fato de que o conhecimento é o principal fator de motivação mencionado pelas empresas, mas, paradoxalmente, a principal atividade das parcerias são atividades de desenvolvimento experimental. Os projetos de desenvolvimento experimental são projetos de aplicação do conhecimento em produtos 
e processos novos ou melhoria dos antigos. Em geral, são projetos de curta duração e não obrigatoriamente geram conhecimento novo, ainda que a literatura (Rosenberg, 1982) aponte vários casos de exceção a esta regra.

$\mathrm{Na}$ presente pesquisa, o uso do conhecimento das universidades apareceu majoritariamente como uma consultoria às atividades analíticas e de testes a serem desenvolvidos pelas empresas, bem como em relação ao uso dos equipamentos para realizar os testes, habilidade que na maioria das vezes falta às empresas. Na percepção dos pesquisadores das universidades, os principais fatores de motivação para colaboração com as empresas são a possibilidade de ver a aplicação de sua pesquisa chegar à sociedade e a disponibilidade de recursos extras para a pesquisa. Segundo o pesquisador de uma ICT do ERJ, "essas teses devem ter um desdobramento social, podem ser usadas para outras pesquisas teóricas, sem dúvida, mas [uma tese] será útil quando entrar na sociedade. Enquanto o ser humano não se beneficiar daquele conhecimento, ela é teórica" (PES13_2012). De um lado e de outro parece que existem razões fortes para explicar o aparente paradoxo entre a visão de que a principal contribuição da universidade é o conhecimento e de que a sua principal contribuição é de fato mais próxima da solução de problemas práticos.

No entanto, o número de obstáculos presente no relacionamento é tão grande que acaba gerando desestímulos a essa interação. O principal problema consiste na burocracia das universidades, uma característica marcante dos órgãos públicos brasileiros. Apesar do forte estímulo a essas parcerias, pouco foi feito pelo governo para tornar os atores aptos para interagirem. Além disso, a necessidade de se criarem os NITs imposta pela Lei de Inovação (10.973/2004) foi feita de forma atabalhoada, uma vez que não foram dadas condições adequadas para as universidades, como, por exemplo, disponibilização de auxílio financeiro para contratação de pessoal especializado e capacitado. A falta de planejamento criou grandes entraves à aproximação entre empresas e universidades e ampliou as dificuldades que normalmente estão presentes neste relacionamento.

O segundo obstáculo mencionado foi um conjunto de fatores intrínseco ao relacionamento empresa-universidade - a desconfiança, o distanciamento e a falta de diálogo existente entre pesquisadores e empresas. Em sistemas de inovação mais desenvolvidos e com maior experiência no relacionamento empresa-universidade, estes obstáculos podem ser reduzidos, mas estarão sempre presentes devido às diferenças de ambiente, tempo, objetivo e visão das empresas e universidades. 
O terceiro obstáculo presente é a dificuldade de negociação da propriedade intelectual no desenvolvimento de pesquisa conjunta e prestação de serviços. A principal razão encontrada para esta dificuldade está na alta expectativa das universidades em conseguirem uma grande quantidade de recursos extras com os licenciamentos. As dificuldades com o escalonamento da pesquisa científica foram também citadas pelos entrevistados como um importante obstáculo à interação empresauniversidade. Relacionada a este problema também está a falta de calibragem dos equipamentos das universidades, outro obstáculo à interação. Em ambos os casos há uma cobrança exacerbada sobre as universidades, que não têm obrigação de realizar escalonamento, nem ter equipamentos calibrados, pois têm um foco maior no aprendizado e em pesquisas que não requerem estas exigências. Porém, esta cobrança ocorre devido à falta de uma malha de pequenas empresas de base tecnológica que possa realizar tais atividades para as empresas e sirvam assim como uma ponte na parceria do setor empresarial com o acadêmico. ${ }^{9}$

Entre os pesquisadores, um obstáculo bastante importante foram as dificuldades encontradas pela gestão familiar das empresas brasileiras, o que resulta em baixos investimentos em inovação, em inexistência de estrutura interna capaz de colaborar com as universidades, falta de pessoal qualificado em pesquisa e desenvolvimento (P\&D) e falta de visão de longo prazo da pesquisa. Acredita-se que somente o desenvolvimento do setor e a profissionalização da gestão nas empresas permitirão superar este tipo de obstáculo.

Percebe-se que os obstáculos presentes na interação empresa-universidade no sistema farmacêutico de inovação brasileiro podem ser sanados pela existência de atores capazes de fazer a ligação da pesquisa de bancada para escalas maiores, bem como pela existência de orçamento de pesquisa nas universidades e suas melhores estruturações, regras, procedimentos e menores burocracias. Os problemas ficam maiores, porque, além dos obstáculos intrínsecos ao relacionamento empresa-universidade, muitos outros também estão presentes dificultando ainda mais a interação. Acredita-se, porém, que com a ampliação dos casos e das experiências estes problemas poderão diminuir.

Independentemente do maior ou menor número de obstáculos, os entrevistados declararam que a principal forma de resolvê-los é ser persistente, flexível, buscar compreender o ponto de vista do parceiro e negociar caso a caso. Alguns fatores, como

\footnotetext{
${ }^{9}$ Para mais detalhes desta discussão, ver Paranhos (2012).
} 
relacionamento pessoal entre pessoas das empresas e pesquisadores e funcionários das universidades, podem facilitar e agilizar o processo burocrático. Além disso, percepção pelo governo da importância da inovação para que este estabeleça um marco regulatório e programas de fomento que estimulem o relacionamento empresa-universidade e a inovação; interesse pelo projeto e reconhecimento adquirido ao se interagir com empresas; e o conhecimento sobre o meio empresarial e industrial adquirido pelos alunos e pesquisadores dos grupos de pesquisa que interagem com empresas também foram mencionados como importantes fatores para viabilização do relacionamento empresa-universidade. Os entrevistados mencionaram que, na presença de profissionais nas empresas com estas informações sobre o meio acadêmico, o relacionamento pode ser mais harmônico e com menores atritos em seu desenvolvimento.

Os NITs das universidades têm sido um importante fator facilitador deste processo. Eles têm se mostrado muito relevantes na interlocução entre empresas e pesquisadores e no estabelecimento de rotinas para estabelecer contato, formular contratos e acompanhar projetos. Apesar das dificuldades no desenvolvimento e estruturação destes núcleos, as opiniões sobre sua atuação são bastante positivas ainda que seu potencial não esteja sendo totalmente utilizado. Pelo lado dos pesquisadores, são auxiliados em diversas atividades, em especial, no depósito de patentes (anteriormente tinham que aprender e fazer sozinhos), no estabelecimento inicial dos contatos e na formulação dos contratos de parceria e de direitos de propriedade intelectual, como mostra o relato de um pesquisador no ERJ: "Eu vejo que [o NIT] avançou muito. Já tem um quadro de pessoal adequado, com advogados etc. Porém não há mais avanço, pois os brasileiros ainda não reconhecem a importância de proteger o capital intelectual nacional” (PES1_2008).

O estabelecimento de rotinas de atuação dos NITs parece constituir uma forma de estabelecimento das próprias regras que facilitam a divulgação da pesquisa universitária ao setor empresarial, assim como a aproximação do setor empresarial com a universidade, ajudando na identificação do parceiro ideal e na redução da diferença de expectativas de empresas e pesquisadores. As atividades de divulgação da pesquisa acadêmica, de criação de redes, de estabelecimento de contratos, de montagem dos projetos e gestão da parceria mostram-se bastante eficazes e positivas no desenvolvimento do relacionamento empresa-universidade.

Diversos destes problemas e obstáculos enfrentados por empresas e universidades ocorrem porque o governo, embora tenha estabelecido o relacionamento 
empresa-universidade como foco de suas estratégias para estímulo à geração de inovação no país, iniciou suas ações de forma prematura, sem análise prévia das questões e necessidades relacionadas, focando principalmente no financiamento da pesquisa acadêmica em parceria com o setor empresarial. Mesmo com suas estratégias desordenadas, o governo hoje tem tido um importante papel de financiador junto às empresas nacionais que têm portes moderados e ainda disponibilizam poucos recursos para investimentos em inovação. De acordo com as empresas no Brasil, o financiamento do governo aumentou sua capacidade de realizar mais projetos em parceria, funcionando como um catalisador deste processo de aproximação com as universidades. Grande parte das empresas entrevistadas já recebeu ou recebe recursos públicos para financiamento de parcerias.

Apesar da relevância do financiamento do governo no Brasil, muitas críticas foram feitas aos programas e editais voltados para este fim, principalmente em relação ao maior direcionamento dos recursos para as universidades, ao pequeno prazo disponibilizado para desenvolvimento da parceria (média de dois anos - pouco tempo para pesquisas neste setor, que duram em média dez anos) e à pulverização de recursos em diversos temas, sem foco específico, além de outros problemas relacionados ao processo de avaliação e seleção dos projetos submetidos e à burocracia na submissão e execução dos projetos.

No entanto, ações do governo e estímulos à interação empresa-universidade não são os únicos fatores necessários para promover o relacionamento e a geração de inovação. O setor farmacêutico é muito complexo e depende do estabelecimento de instituições governamentais bem desenvolvidas para trabalhar de forma adequada. Aspectos regulatórios e direitos de propriedade intelectual são bastante significativos para a produção e a inovação neste setor. Por esta razão, em conjunto com os atuais estímulos do governo para o desenvolvimento do setor farmacêutico no Brasil, é mandatória a criação de um marco regulatório e a melhoria da eficiência e agilidade das agências reguladoras, das instituições governamentais e das universidades públicas. Estes fatores contribuirão para reduzir a incerteza e aumentar a propensão das empresas em assumir riscos e investir em produção e inovação.

No ERJ, em particular, alguns obstáculos foram listados como significativos no impedimento ao desenvolvimento do sistema farmacêutico de inovação; entre os mais comentados estão a alta tributação, principalmente do ICMS, a ausência de políticas de apoio do governo estadual e o forte desenvolvimento do setor no ESP. O último ponto 
foi comentado por um entrevistado de uma empresa de pesquisa farmacêutica: "As desvantagens são óbvias. A economia não está no Rio de Janeiro, está em São Paulo. Isso aí já é um grande problema, apesar de todo esse conhecimento" (EPF3_2011). O comentário diz respeito à grande importância da economia paulista para o país e da importância do setor farmacêutico neste estado. O entrevistado de uma empresa farmacêutica nacional e um ator ligado ao setor enfatizaram a questão da guerra físcal entre os estados, da alta tributação e da ausência de políticas efetivas para o setor. $\mathrm{O}$ entrevistado da empresa farmacêutica não tinha qualquer conhecimento sobre os decretos (citados acima) que preveem incentivos fiscais à cadeia produtiva farmacêutica. O segundo relato mostra que tais decretos parecem não gerar muitos efeitos virtuosos.

"[...] foi o que citei anteriormente, a parte tributária. Acho que o Rio de Janeiro como coletor de impostos está à frente de todos os estados, é um dos que têm a carga tributária mais alta. As outras desvantagens são mais comuns aos outros estados [...]. Voltado para a indústria farmacêutica, o apoio do estado, eu considero nulo. Não identifico nenhum apoio por parte do estado, tanto na parte de tributação, quanto na parte de estruturação. Qualquer favorecimento eu [pessoalmente] não consigo identificar que o Estado tome ações ou providências voltadas para este fim" (EFN11_2011).

“[...] a desvantagem de ICMS para produção e distribuição. [...] Goiás apresentou uma alternativa para as indústrias [...] [localizadas aqui], para ir para lá com vantagens fiscais enormes. Os grandes distribuidores que tinham no ERJ foram minguando e ficaram um ou dois. [...] [Os Decretos de incentivos fiscais não servem] para atrair novos investimentos, [mas] pelo menos para ajudar os que estão aqui sim. [...] Eu acho que eles fizeram algumas medidas para ajudar um pouco, mas é tímido ainda. O problema da carga tributária sobre medicamentos que ainda é muito alto, aqui no RJ o ICMS continua alto para a indústria farmacêutica e é um fator fundamental hoje quando se discute carga tributária. Se o Rio tivesse uma ação mais pró-ativa sobre o ICMS, aí realmente muita gente ia querer vir para cá" (OAS7_2012).

Não obstante, foram citadas também vantagens do ERJ frente aos demais estados do país. Entre as principais, estão a existência de diversos centros de excelência acadêmica e científica, o que leva a uma alta densidade demográfica de pesquisadores; o polo de Jacarepaguá que gera mão de obra qualificada; o porto e o aeroporto para entrada e saída de mercadorias; a proximidade com clientes, ainda que os fornecedores estejam em São Paulo, pela facilidade nas vendas; e o bom momento de atração de 
investimentos que o ERJ passa, conforme o comentário do entrevistado da empresa de pesquisa farmacêutica abaixo. No entanto, como expõe o pesquisador de uma ICT do ERJ a seguir, a ligação mais densa entre ICTs e empresas ainda não ocorreu devido à falta de apoio do governo. Por outro lado, o ator ligado ao setor comenta sobre a perda de profissionais para outros estados: “[...] infelizmente no ERJ não temos nada disso, esses laboratórios todos que eu citei para você são do ESP, nenhum deles é do Rio. [...] posso te dizer de vários pesquisadores cariocas que estão trabalhando para [esta empresa em SP]" (OAS7_2012).

"Eu acho que a gente tem uma capacidade intelectual no estado que é muito grande. Disso eu não tenho dúvidas, [...] [esta universidade] é um polo de inteligência que, apesar de todos os problemas de infra[estrutura], tem muita gente boa. Isso é uma vantagem. É obvio que esse momento que a gente está vivendo no Rio de Janeiro também é uma vantagem em termos de visibilidade e de investimentos. É obvio que daqui pra frente com esse virtuosismo e com esse crescimento certamente vai ter mais gente querendo colocar dinheiro aqui. Então, isso é bom" (EPF3_2011).

"Se você olhar dez anos e agora, é completamente diferente, o governo está muito mais sensível [à parceria ICT-empresa]. Mas eu acho que a gente ainda não está sabendo 'marcar o gol'. Nós estamos fazendo tudo certo, mas a finalização a gente não está sabendo fazer. Não está sabendo fazer porque a gente não tem esse incentivo de recurso" (PES14_2012).

Percebe-se que, no ERJ, a grande quantidade de pesquisadores e ICTs, e as demais vantagens citadas acima, não têm sido suficientes para atrair, por si só, empresas e dinamizar o setor farmacêutico no estado. Isto porque há obstáculos relacionados à carga tributária e ao baixo dinamismo econômico, que estão gerando efeitos negativos sobre o desenvolvimento do setor. É patente a necessidade de políticas industriais e de inovação no ERJ para o apoio ao desenvolvimento do setor e o estímulo às parcerias entre empresas e ICTs. 


\section{Conclusões e implicações de política}

Apesar da excelência acadêmica do ERJ aparecer como uma grande vantagem para o setor farmacêutico no estado, esta não tem sido suficiente para a promoção da interação empresa-universidade. Isto porque o governo estadual demorou muito para promover ações concretas de promoção da capacidade produtiva e inovativa das empresas farmacêuticas. A criação do GECIV-RJ e as primeiras ações que este vem tomando demonstram que o cenário tenderá a sofrer mudanças nos próximos anos com fortalecimento do setor farmacêutico e consequentemente ampliação das interações com as universidades do estado.

Com base na análise da literatura, dos dados secundários e da pesquisa de campo, à guisa de conclusão, pode-se resumir cinco pontos principais que devem ser destacados no sentido de incrementar as relações entre empresas e universidades: 1) a necessidade de fortalecer a estrutura interna de $P \& D$ nas empresas farmacêuticas para a geração de inovação e para melhorar a interação com o setor acadêmico; 2) a necessidade de reestruturar e modernizar a estrutura interna das universidades; 3) a necessidade de um planejamento prévio das ações do governo e da criação de um ambiente propício para a inovação; 4) o papel fundamental desempenhado pelo financiamento do governo e apoio às atividades inovadoras das empresas e para os NITs das universidades, ainda que apenas em sua fase inicial; e 5) a necessidade de regulação e regulamentação eficiente do setor farmacêutico. No sistema farmacêutico de inovação fluminense, em particular, ressalta-se: 1) a necessidade de revisão da carga tributária sobre a produção e distribuição; e 2) o estabelecimento de políticas industriais e de inovação a nível estadual principalmente para fortalecer a infraestrutura tecnológica capaz de fomentar uma melhor interação entre empresa-universidade.

Em suma, as empresas farmacêuticas e as universidades são atores com forte potencial para interagir e desenvolver inovações, mas esta interação não é espontânea, ela precisa ser promovida e estimulada. $\mathrm{O}$ ator mais importante para desenvolvimento de uma política industrial e tecnológica é o governo, devido a sua potencialidade de propulsor ou bloqueador do desenvolvimento de um setor.

Em termos de lições que o estudo aponta para o governo estadual, listam-se duas. Primeiro, o governo estadual deve atuar junto ao governo federal por melhorias na 
atuação de determinados órgãos federais com maior efeito sobre o sistema farmacêutico de inovação para que se amplie a articulação entre estes órgãos, gerando maior aproveitamento das ações e melhoria do ambiente inovativo.

Segundo, cabe ao governo estadual criar um ambiente local propício à inovação no estado. Há ações bastante significativas que podem ser tomadas para ampliar o estímulo à inovação e à interação empresa-universidade no ERJ. A criação do GECIVRJ apresenta grande potencial neste sentido, pois engloba diversos atores ligados ao setor no estado e vem buscado ampliar a articulação entre eles. Além disso, vem realizando estudos para um melhor mapeamento das potencialidades do Rio de Janeiro para o desenvolvimento do setor farmacêutico.

\section{Referências bibliográficas}

BERMUDEZ, J.; EPSZTEJN, R.; OLIVEIRA, M. A.; HASENCLEVER, L. O Acordo TRIPS da OMC e a Proteção Patentária no Brasil: mudanças recentes e implicações para a produção local e o acesso aos medicamentos. Rio de Janeiro: Fiocruz/ENSP, 2000.

BRITTO, J.; BITTENCOURT, P.; CRUZ, W. Interação infraestrutura de ciência e tecnologia (C\&T) e o setor produtivo no Estado do Rio de Janeiro. In: SUZIGAN, W.; ALBUQUERQUE, E.; CARIO, S. Em busca da inovação: interação universidade-empresa no Brasil. São Paulo: Editora Autêntica, 2011. pp.109-157.

BUSS, P.; CARVALHEIRO, J.; CASAS, C. (Orgs.). Medicamentos no Brasil: inovação e acesso. Rio de Janeiro: Editora Fiocruz, 2008.

CAPES - Coordenação de Aperfeiçoamento de Pessoal de Nível Superior. Estatísticas: Sistema Geocapes 2011. Disponível em: www.capes.gov.br (Acesso em 10 Ago. 2012).

CNPq - Conselho Nacional de Desenvolvimento Científico e Tecnológico. Plano Tabular 2010. Disponível em: www.cnpq.br (Acesso em 07 Jul. 2012).

COOKE, P.; URANGA, M.; ETXEBARRIA, G. Regional innovation systems: Institutional and organisational dimensions. Research Policy, v. 26, issues 4-5, pp. 475-491, Inglaterra, Dec. 1997.

FAURÉ, Yves-A.; HASENCLEVER, Lia (Orgs.). Caleidoscópio do desenvolvimento local no Brasil: diversidade das abordagens e das experiências. Rio de Janeiro: EPapers, 2007. 
O desenvolvimento local no Estado do Rio de Janeiro: estudos avançados nas realidades municipais. Rio de Janeiro: E-Papers, 2005.

HASENCLEVER, L. (Coord.) Interação entre instituições de ciência e tecnologia e empresas no sistema farmacêutico de inovação brasileiro: lições para o desenvolvimento do setor no Estado do Rio de Janeiro. Relatório de Pesquisa. Rio de Janeiro: Faperj/UFRJ/Instituto de Economia, 2012.

. Diagnóstico da Indústria Farmacêutica Brasileira. Relatório de pesquisa. Rio de Janeiro: UNESCO/UFRJ/Instituto de Economia, 2002.

HASENCLEVER, L., PARANHOS, J., TORRES, R. Desempenho econômico do Rio de Janeiro: trajetórias passadas e perspectivas futuras. DADOS - Revista de Ciências Sociais, Rio de Janeiro, v. 55, n. 3, pp. 681 a 711, 2012.

IBGE - Instituto Brasileiro de Geografia e Estatística. Pesquisa Industrial Mensal Produção Física, 1996-2010. Rio de Janeiro: IBGE, 2011.

LESSA, C. O Rio de todos os Brasis. 3 ed. Rio de Janeiro: Ed. Record, 2000. pp. 345412.

LUNDVALL, B.-Å., JOHNSON, B., ANDERSEN, E., DALUM, B. National systems of production, innovation and competence building. Research Policy, Inglaterra, v. 31, pp. 213-231, 2002.

MEC - Ministério da Educação. Sistema e-MEC 2012. Disponível em: www.mec.gov.br (Acesso em 10 Ago. 2012).

MTE - Ministério do Trabalho e Emprego. Relação Anual de Informações Sociais, 1996-2009. Rio de Janeiro: MTE, 2010. Disponível em: http://www.mte.gov.br/.

PARANHOS, J. Interação entre empresas e instituições de ciência e tecnologia: o caso do sistema farmacêutico de inovação brasileiro. Rio de Janeiro: Eduerj, 2012.

O sistema farmacêutico de inovação fluminense: forças, fraquezas e desafios ao desenvolvimento. Projeto de Pesquisa submetido ao edital APQ1 da Faperj. Rio de Janeiro: UFRJ/Instituto de Economia, 2011. (Aprovado para financiamento em 2012)

ROSENBERG, N. Inside the black box: technology and economics. Cambridge: Cambridge University Press, 1982.

SELAN, B.; KANNEBLEY JÚNIOR, S.; PORTO, G. S. Relatório setorial sobre inovação tecnológica na indústria farmacêutica brasileira: uma análise a partir dos indicadores de inovação. Ribeirão Preto: Fipase, 2007. 79 p.

SUZIGAN, W.; ALBUQUERQUE, E. A interação entre universidades e empresas em perspectiva histórica no Brasil. In: SUZIGAN, W.; ALBUQUERQUE, E.; 
CARIO, S. Em busca da inovação: interação universidade-empresa no Brasil. São Paulo: Editora Autêntica, 2011. pp.17-43.

URANI, A; GIAMBAGI, F. (Orgs.). Rio: A Hora da Virada. Rio de Janeiro: Campus/Elsevier, 2011.

\section{Decretos e Leis:}

Decreto do Estado do Rio de Janeiro:

- $\quad$ n $^{\circ} 24.857$ de 26 de novembro de 1998.

- $\quad \mathrm{n}^{\circ} 36.450$ de 29 de outubro de 2004.

- $\mathrm{n}^{\circ} 42.302$ de 12 de fevereiro de 2010.

- no $^{\circ} 43.315$ de 25 de novembro de 2011.

Lei do Estado do Rio de Janeiro:

- $\quad \mathrm{n}^{\mathrm{o}} 5.361$ de 29 de dezembro de 2008.

Lei Federal:

- $\quad \mathrm{N}^{\mathrm{o}} 10.973$ de 02 de dezembro de 2004. 